\title{
Enhanced Nonlinear Generation in a Three-Level Medium with Spatially Dependent Coherence
}

\author{
E. Paspalakis \\ Materials Science Department, School of Natural Sciences, University of Patras, Patras 265 04, Greece \\ Z. Kis \\ Research Institute for Solid State Physics and Optics, P.O. Box 49, H-1525 Budapest, Hungary
}

(Dated: October 17, 2018)

\begin{abstract}
We consider a method for efficient parametric generation of a laser pulse. A single laser field is injected to a three-level medium which has two lower states and one excited state. The lower states are prepared initially in a position-dependent coherent superposition state. It is shown that by proper choice of the position dependence of the superposition along the direction of propagation, the incoming field can be converted completely to a new field.
\end{abstract}

The problem of resonant nonlinear optics with high efficiency using phase-coherent media has attracted a lot of interest recently 1]. The usual systems used for this purpose are the three-level $\Lambda$-type system 2, 3, 4, 5, 6], and various configurations of multi-level systems [7, 8, 9, 10]. Some very interesting experiments using these systems have been performed 1, 11, 12, 13, 14, 15], demonstrating the potential for applications of these phenomena. In a similar area of studies, Grobe and co-workers have shown that a dielectric medium with initial spatial excitation can have several novel properties [16]. In this work, we study the potential for nonlinear conversion between two laser pulses using a $\Lambda$-type system with initial spatial excitation. We show that the existence of a spatially dependent coherence can lead to parametric generation of a new laser pulse with unity conversion efficiency.

The quantum system under consideration is shown in Fig. 11 The spatio-temporal dynamics of the system can be described by the coupled Maxwell-Schrödinger equations in the rotating wave, dipole, and slowly varying envelope approximations

$$
\begin{aligned}
i \frac{\partial}{\partial \tau} \boldsymbol{b}(\zeta, \tau) & =\boldsymbol{H}(\zeta, \tau) \boldsymbol{b}(\zeta, \tau) \\
\frac{\partial}{\partial \zeta} \Omega_{n}(\zeta, \tau) & =i a_{n} b_{n}(\zeta, \tau) b_{0}^{*}(\zeta, \tau), \quad n=1,2
\end{aligned}
$$

with

$$
\boldsymbol{H}(\zeta, \tau)=\left[\begin{array}{ccc}
\delta_{1}-i \frac{\gamma}{2} & \Omega_{1}^{*}(\zeta, \tau) & \Omega_{2}^{*}(\zeta, \tau) \\
\Omega_{1}(\zeta, \tau) & 0 & 0 \\
\Omega_{2}(\zeta, \tau) & 0 & \delta_{1}-\delta_{2}
\end{array}\right]
$$

and $\boldsymbol{b}(\zeta, \tau)=\left[b_{0}(\zeta, \tau), b_{1}(\zeta, \tau), b_{2}(\zeta, \tau)\right]^{T}$. These equations are written in the local frame where $\zeta=z$ and $\tau=t-z / c$. Here, $\Omega_{n}(\zeta, \tau)$ are the Rabi frequencies and $\delta_{n}$ are the laser field detunings from resonance. Also, $\gamma$ denotes the decay rate of the excited state out of the system and $a_{n}$ the propagation constant.

We assume that the system is initially prepared in a superposition of the lower levels, i.e. we assume a phaseonium medium which was first proposed by Scully [17], such that

$$
|\psi(\zeta, \tau=0)\rangle=b_{1}(\zeta)|1\rangle+b_{2}(\zeta)|2\rangle,
$$

with $b_{1}(\zeta)$ and $b_{2}(\zeta)$ being, in general, complex satisfying $\left|b_{1}(\zeta)\right|^{2}+\left|b_{2}(\zeta)\right|^{2}=1$. As it has been analyzed in detail by Csesznegi et al. [16] any desired coherent superposition of such type can be created with the use of stimulated Raman adiabatic passage (STIRAP) [18]. This technique uses two laser pulses, ordered in a counterintuitive sequence, that are applied in the preparation stage to the medium. The shape of these pulses [16] will determine the specific form of $b_{1}(\zeta)$ and $b_{2}(\zeta)$. We also assume that the two-photon resonance condition, $\delta_{1}=\delta_{2}=\delta$, is satisfied. If the excited state $|0\rangle$ decays rapidly and the laser-matter interaction is weak, so that the following relations $\left|\Omega_{n}\right| \ll \gamma, \gamma \bar{\tau} \gg 1,\left|\Omega_{n}\right|^{2} \bar{\tau} \ll \gamma$ are satisfied, with $\bar{\tau}$ being a characteristic pulse length, the approximate solution of Eq. (1a) is 2, 10]

$$
\begin{aligned}
& b_{1}(\zeta, \tau) \approx b_{1}(\zeta), \quad b_{2}(\zeta, \tau) \approx b_{2}(\zeta) \\
& b_{0}(\zeta, \tau) \approx-\frac{\Omega_{1}^{*}(\zeta, \tau) b_{1}(\zeta)+\Omega_{2}^{*}(\zeta, \tau) b_{2}(\zeta)}{\delta-i \gamma / 2}
\end{aligned}
$$

and the propagation equation for the laser fields, Eqs. (1b), reduce to

$$
\frac{\partial}{\partial \zeta} \boldsymbol{\Omega}(\zeta, \tau)=-i \boldsymbol{K}(\zeta) \boldsymbol{\Omega}(\zeta, \tau)
$$

with

$$
\boldsymbol{K}(\zeta)=\left[\begin{array}{cc}
\alpha_{1}\left|b_{1}(\zeta)\right|^{2} & \alpha_{1} b_{1}(\zeta) b_{2}^{*}(\zeta) \\
\alpha_{2} b_{2}(\zeta) b_{1}^{*}(\zeta) & \alpha_{2}\left|b_{2}(\zeta)\right|^{2}
\end{array}\right]
$$

Here, $\alpha_{n}=a_{n} /(\delta+i \gamma / 2)$ and the vector of the Rabi frequencies is given by $\Omega(\zeta, \tau)=\left[\Omega_{1}(\zeta, \tau), \Omega_{2}(\zeta, \tau)\right]^{T}$.

In this work we restrict the discussion to the case when the propagation constants are equal $a_{1}=a_{2}$, which leads to $\alpha_{1}=\alpha_{2}=\alpha$. However, our results apply also to the case that $a_{1} \neq a_{2}$, which will be studied in detail elsewhere. As we are interested in the phenomenon of parametric generation in our system we will take the initial conditions as $\Omega_{1}(\zeta=0, \tau)=\Omega(\tau)$ and $\Omega_{2}(\zeta=0, \tau)=0$. In the case that the probability amplitudes of the phaseonium are independent of $\zeta$, i.e. $b_{1}(\zeta)=b_{1}, b_{2}(\zeta)=b_{2}$, 
then the solution of Eq. (5) is given by [2]

$$
\begin{aligned}
& \Omega_{1}(\zeta, \tau)=\left(\left|b_{1}\right|^{2} e^{-i \alpha \zeta}+\left|b_{2}\right|^{2}\right) \Omega(\tau), \\
& \Omega_{2}(\zeta, \tau)=b_{1}^{*} b_{2}\left(-1+e^{-i \alpha \zeta}\right) \Omega(\tau) .
\end{aligned}
$$

The long distance result, which is mathematically obtained in the limit $\zeta \rightarrow \infty$ and in practice it means $\zeta \gg|1 / \operatorname{Im}(\alpha)|$, is independent of $\alpha$, as $\Omega_{1}(\zeta \rightarrow \infty, \tau)=$ $\left|b_{2}\right|^{2} \Omega(\tau)$ and $\Omega_{2}(\zeta \rightarrow \infty, \tau)=-b_{1}^{*} b_{2} \Omega(\tau)$. This means that complete conversion is not possible in this case.

The results are very different in the case that $b_{1}(\zeta)$, $b_{2}(\zeta)$ are not constant. First of all, there is no general analytic solution of the propagation equation (5). This equation resembles Schrödinger equation with the replacement $\zeta \leftrightarrow t$, where the propagator $\boldsymbol{K}(\zeta)$, Eq. (6), plays the role of the Hamiltonian and is non-Hermitian in this case. In the case of a time-dependent Hamiltonian general solutions can be obtained if the dynamics satisfies adiabaticity [19]. Therefore, we study the adiabatic evolution of the system [20].

The adiabatic evolution is succeeded if the condition

$$
|v(\zeta)| \ll|\alpha|
$$

is fulfilled, with

$$
v(\zeta)=i\left(b_{1}(\zeta) \frac{\partial b_{2}(\zeta)}{\partial \zeta}-\frac{\partial b_{1}(\zeta)}{\partial \zeta} b_{2}(\zeta)\right)
$$

These two equations show that in the adiabatic limit the spatial variation of the probability amplitudes should be smooth and their rate of change to be smaller than $|\alpha|$. The adiabatic solutions of Eq. (5) is then given by

$$
\begin{aligned}
& \Omega_{1}(\zeta, \tau)=\left[b_{2}(0) b_{2}(\zeta)^{*}+e^{-i \alpha \zeta} b_{1}(0)^{*} b_{1}(\zeta)\right] \Omega(\tau)(10 \mathrm{a}) \\
& \left.\Omega_{2}(\zeta, \tau)=\left[-b_{2}(0) b_{1}(\zeta)^{*}+e^{-i \alpha \zeta} b_{1}(0)^{*} b_{2}(\zeta)\right] \Omega((\tau)) 0 \mathrm{~b}\right)
\end{aligned}
$$

If we choose $b_{1}(0)=0, b_{2}(0)=e^{i \phi_{2}}$ and $b_{1}(\zeta \rightarrow \infty)=$ $e^{i \phi_{1}}, b_{2}(\zeta \rightarrow \infty)=0$, then in the adiabatic limit

$$
\Omega_{1}(\zeta \rightarrow \infty, \tau)=0, \Omega_{2}(\zeta \rightarrow \infty, \tau)=-e^{i\left(\phi_{2}-\phi_{1}\right)} \Omega(\tau)
$$

which means that $\left|\Omega_{2}(\zeta \rightarrow \infty, \tau)\right|^{2}=|\Omega(\tau)|^{2}$. Therefore, by proper choice of the initial spatial excitation of the system complete nonlinear conversion is possible between the two laser fields. One may notice that the choice of the initial distribution is counterintuitive: we need at the entrance of the medium $b_{1}(\zeta)=0$ and in the long distance limit $b_{2}(\zeta)=0$. We note that, in addition to the conditions specified above, the spatial variation of the amplitudes should satisfy the adiabaticity condition Eq. (8), as only in this case the solutions (10a) and (10b) are valid.
The process of parametric generation in our system is illustrated in Fig. [2] where the spatio-temporal evolution of the normalized intensities of the laser pulses is shown. The results have been obtained from a numerical solution of Eq. (5). The initial spatial distributions are chosen as

$$
\begin{aligned}
& b_{1}(\zeta)=\sqrt{\frac{1}{1+e^{-\left(\zeta-\zeta_{0}\right) / \bar{\zeta}}}} \\
& b_{2}(\zeta)=\sqrt{\frac{1}{1+e^{\left(\zeta-\zeta_{0}\right) / \bar{\zeta}}}}
\end{aligned}
$$

and the incoming pulse has a sin-squared shape. It is clear that the incoming laser pulse is completely converted to a new laser pulse. The accuracy of the approximations that lead to Eq. (5) has been assessed by comparing with the numerical solution of Eqs. (1a) and (1b). The agreement between the two results is very good, implying that our approximations are valid for the chosen parameter set.

In this paper, we have investigated resonant nonlinear optical processes that occur when a medium is initially prepared in a spatially dependent coherent superposition. In particular, for a $\Lambda$-type system we have demonstrated that by proper choice of the spatially dependent initial excitation complete nonlinear conversion between two laser pulses is possible. An interesting application of the present scheme is a coherent polarization rotator. Let us consider a $\Lambda$ scheme with lower states $J_{g}=1$, $M_{g}= \pm 1$ and excited state $J_{e}=0$. Then, if the injected field is left-hand circularly polarized, in the medium it will be transferred smoothly to the other mode and a right-hand circularly polarized light will exit the medium.

We note before closing that the process studied in this work is the spatial analog of STIRAP. That is, by using STIRAP in a $\Lambda$-type system complete population transfer from one state to another state is possible by proper choice of the Rabi frequencies. In our method complete conversion from one laser pulse to another laser pulse is possible by proper choice of the initial spatial excitation of the medium. Both methods are based on a counterintuitive excitation and on the adiabatic evolution of the system.

E.P. would like to thank N.J. Kylstra for assistance in the numerical simulations. This work was supported by the European Union Research and Training Network COCOMO, contract HPRN-CT-1999-00129. Z.K. acknowledges the support of the János Bolyai program of the Hungarian Academy of Sciences.
[1] M.D. Lukin, P.R. Hemmer and M.O. Scully, Adv. Atom. Mol. Opt. Phys. 42, 347 (2000).
[2] J.H. Eberly, A. Rahman and R. Grobe, Phys. Rev. Lett. 76, 3687 (1996). 
[3] S.E. Harris and M. Jain, Opt. Lett. 22, 636 (1997).

[4] W. Harshawardan and G.S. Agarwal, Phys. Rev. A 58, 598 (1998).

[5] L. Deng, M.G. Payne and W.R. Garrett, Phys. Rev. A 58, 707 (1998).

[6] S.H. Choi and G. Vemuri, Opt. Commun. 153, 257 (1998).

[7] M.D. Lukin, P.R. Hemmer, M. Löffler and M.O. Scully, Phys. Rev. Lett. 81, 2675 (1998).

[8] E.A. Korsunsky and D.V. Kosachiov, Phys. Rev. A 60, 4996 (1999).

[9] R.W. Boyd and M.O. Scully, Appl. Phys. Lett. 77, 3559 (2000).

[10] E. Paspalakis and P.L. Knight, J. Mod. Opt. 49, 87 (2002); E. Paspalakis, N.J. Kylstra and P.L. Knight, Phys. Rev. A 65, 053808 (2002).

[11] P.R. Hemmer, D.P. Katz, J. Donoghue, M. CroninGolomb, M.S. Shahriar and P. Kumar, Opt. Lett. 20, 982 (1995).

[12] M. Jain, H. Xia, G.Y. Yin, A.J. Merriam, and S.E. Harris, Phys. Rev. Lett. 77, 4326 (1996).

[13] A.J. Merriam, S.J. Sharpe, H. Xia, D. Manuszak, G.Y. Yin and S.E. Harris, Opt. Lett. 24, 625 (1999).

[14] A.J. Merriam, S.J. Sharpe, M. Shverdin, D. Manuszak, G.Y. Yin and S.E. Harris, Phys. Rev. Lett. 84, 5308 (2000).

[15] A.F. Huss, N. Peer, R. Lammegger, E.A. Korsunsky and L. Windholz, Phys. Rev. A 63, 013802 (2001).

[16] J.R. Csesznegi and R. Grobe, Phys. Rev. Lett. 79, 3162 (1997); J.R. Csesznegi, B.K. Clark and R. Grobe, Phys. Rev. A 57, 4860 (1998).

[17] M.O. Scully, Phys. Rev. Lett. 67, 1855 (1991).

[18] K. Bergmann, H. Theuer and B.W. Shore, Rev. Mod. Phys. 70, 1003, (1998); N.V. Vitanov, M. Fleischhauer, B.W. Shore and K. Bergmann, Adv. Atom. Mol. Opt. Phys. 46, 55 (2001).

[19] A. Messiah, Quantum Mechanics (Dover Pubns, 2000).

[20] Usually adiabatic evolution refers to temporal evolution. Here, we will use it for spatial evolution.

\section{LIST OF FIGURE CAPTIONS}

Fig.1. Schematic diagram of the system studied. The excited state $|0\rangle$ is coupled by two coherent laser pulses to the lower states $|1\rangle$ and $|2\rangle$. In the calculations presented in Fig. $2 \Omega_{1}(\zeta, \tau)$ is the Rabi frequency of the incoming pulse and $\Omega_{2}(\zeta, \tau)$ is the Rabi frequency of the generated pulse.

Fig.2. The normalized field intensities $\left|\Omega_{1}(\zeta, \tau)\right|^{2} /\left|\Omega_{1}\right|^{2}$ for (a), and $\left|\Omega_{2}(\zeta, \tau)\right|^{2} /\left|\Omega_{1}\right|^{2}$ for (b) as a function of $\tau$ for different values of $\zeta$, with $\zeta=0$ (solid curves), $\zeta=100$ (dashed curves) and $\zeta=200$ (dot-dashed curves). In figure (c), we present the maximum of the normalized field intensities as a function of $\zeta$ for the incoming field (dashed curve) and the generated field (solid curve). The incident pulse is $\Omega(\tau)=\Omega_{1} \sin ^{2}\left(\tau \pi / \tau_{p}\right)$, with $0 \leq \tau \leq \tau_{p}$. The parameters used in the calculations are $a_{1}=a_{2}=$ $1000, \Omega_{1}=0.01, \tau_{p}=50, \delta=0, \gamma=100, \zeta_{0}=100$ and $\bar{\zeta}=5$. All quantities are in arbitrary units. 


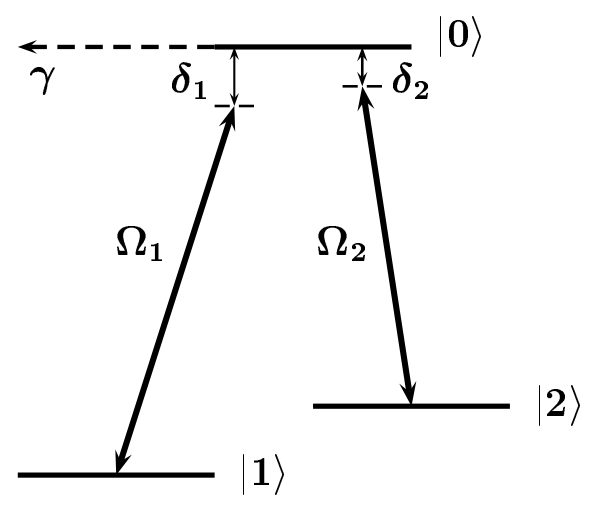

FIG. 1: Paspalakis and Kis 

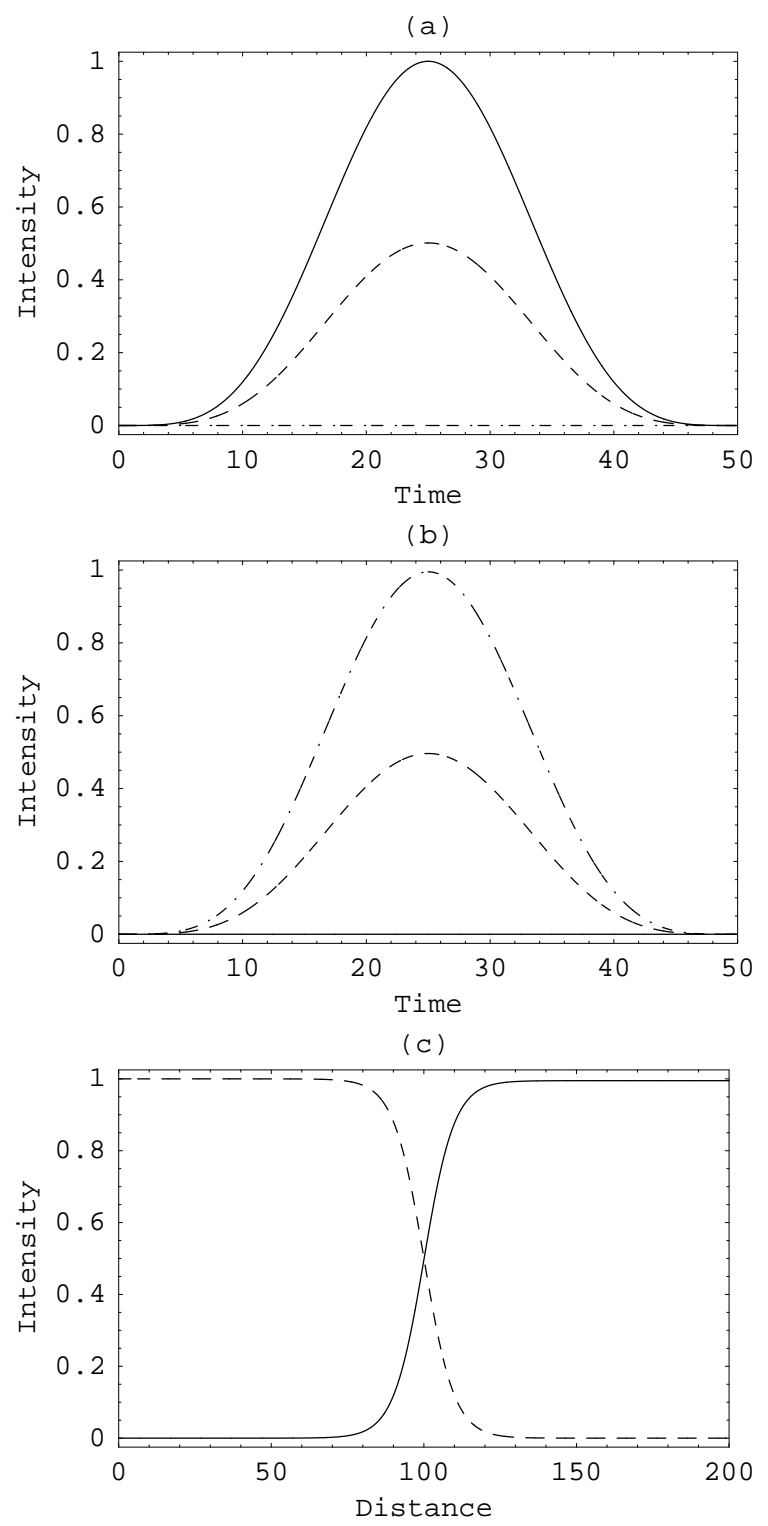

FIG. 2: Paspalakis and Kis 